\title{
Augmentation of donor-derived Tax-specific CTL responses by a novel Tax epitope-specific CD4+ helper T-cells in ATL patients after allogeneic hematopoietic stem cell transplantation
}

\author{
Atsuhiko Hasegawa ${ }^{1 *}$, Yotaro Tamai ${ }^{1}$, Ayako Takamori ${ }^{1}$, Amane Sasada ${ }^{1}$, Ryuji Tanosaki ${ }^{2}$, Ilseung Choi ${ }^{3}$, \\ Atae Utsunomiya ${ }^{4}$, Yasuhiro Maeda ${ }^{5}$, Yoshihisa Yamano ${ }^{6}$, Tetsuya Eto ${ }^{7}, K_{i}$-Ryang Koh ${ }^{8}$, Hirohisa Nakamae ${ }^{8}$, \\ Youko Suehiro ${ }^{3}$, Koji Kato ${ }^{9}$, Shigeki Takemoto ${ }^{10}$, Jun Okamura ${ }^{11}$, Naokuni Uike ${ }^{3}$, Mari Kannagi \\ From 16th International Conference on Human Retroviruses: HTLV and Related Viruses \\ Montreal, Canada. 26-30 June 2013
}

Adult T-cell leukemia/ lymphoma (ATL) is an aggressive $\mathrm{T}$-cell malignancy caused by human $\mathrm{T}$-cell leukemia virus type 1 (HTLV-1) and characterized by extremely poor prognosis because of intrinsic drug resistance to cytotoxic agents. Recently, allogeneic hematopoietic stem cell transplantation (allo-HSCT) has been shown to be an effective treatment for ATL due to graft-versus-leukemia effects. However, donor-derived HTLV-1-specific T-cell responses in ATL patients after allo-HSCT are not well understood. In this study, blood samples from $15 \mathrm{ATL}$ patients at 180 days following allo-HSCT from seronegative donors were screened for IFN-g production of donor-derived T-cells against Tax protein. Among 15 patients, Tax-specific $\mathrm{T}$-cell responses were observed in 11 patients. Direct detection with Tax/MHC class I tetramers revealed that donor-derived Tax-specific $\mathrm{CD}^{+}$cytotoxic T-lymphocytes (CTLs) were present in 12 of 15 patients. We then identified a novel Tax epitope (Tax155-167) presented by HLA-DR1 (HLA-DRB1*0101) and examined the presence of Tax-specific $\mathrm{CD} 4^{+} \mathrm{T}$-cells in $3 \mathrm{HLA}^{-D R} 1^{+}$patients after allo-HSCT using a newly generated Tax155-167/HLA-DR1 tetramer. Tax155-167specific $\mathrm{CD} 4^{+} \mathrm{T}$-cells were found to be present in all HLA-DR $1^{+}$patients tested. Furthermore, in vitro stimulation of PBMCs from HLA-DR1 ${ }^{+}$HLA-A*2402 ${ }^{+}$patients with both Tax155-167 and HLA-A*2402-restricted CTL

\footnotetext{
* Correspondence: hase.impt@tmd.ac.jp

'Department of Immunotherapeutics, Tokyo Medical and Dental University, Tokyo, Japan

Full list of author information is available at the end of the article
}

epitope (Tax301-309) led to robust expansion of Tax301309-specific CTLs. Our results suggest that donor-derived HTLV-1-specific both $\mathrm{CD} 4^{+}$and $\mathrm{CD} 8^{+} \mathrm{T}$-cells could be induced in ATL patients after allo-HSCT from seronegative donors, probably due to exposure to HTLV-1 antigens from recipient-derived ATL cells, and the HTLV-1-specific CD4 ${ }^{+}$T-cells may contribute to efficient induction of donor-derived HTLV-1-specific CTL responses.

\section{Authors' details}

'Department of Immunotherapeutics, Tokyo Medical and Dental University, Tokyo, Japan. ${ }^{2}$ Clinical Laboratories Division, National Cancer Center Hospital, Tokyo, Japan. ${ }^{3}$ Department of Hematology, National Kyushu Cancer Center, Fukuoka, Japan. ${ }^{4}$ Department of Hematology, Imamura Bun-in Hospital, Kagoshima, Japan. ${ }^{5}$ Division of Hematology, Department of Internal Medicine, Kinki University School of Medicine, Osaka, Japan. ${ }^{6}$ Department of Rare Diseases Research, Institute of Medical Science, St. Marianna University Graduate School of Medicine, Kawasaki, Japan. ${ }^{7}$ Department of Hematology, Hamanomachi Hospital, Fukuoka, Japan. ${ }^{8}$ Department of Hematology, Graduate School of Medicine, Osaka City University, Osaka, Japan. ${ }^{9}$ Department of Medicine and Biosystemic Science, Kyushu University Graduate School of Medical Sciences, Fukuoka, Japan. ${ }^{10}$ Department of Hematology, National Hospital Organization Kumamoto Medical Center, Kumamoto, Japan. ${ }^{11}$ Institute for Clinical Research, National Kyushu Cancer Center, Fukuoka, Japan.

Published: 7 January 2014

$$
\begin{aligned}
& \text { doi:10.1186/1742-4690-11-S1-O35 } \\
& \text { Cite this article as: Hasegawa et al.: Augmentation of donor-derived } \\
& \text { Tax-specific CTL responses by a novel Tax epitope-specific CD4+ helper } \\
& \text { T-cells in ATL patients after allogeneic hematopoietic stem cell } \\
& \text { transplantation. Retrovirology } 2014 \text { 11(Suppl 1):O35. }
\end{aligned}
$$

